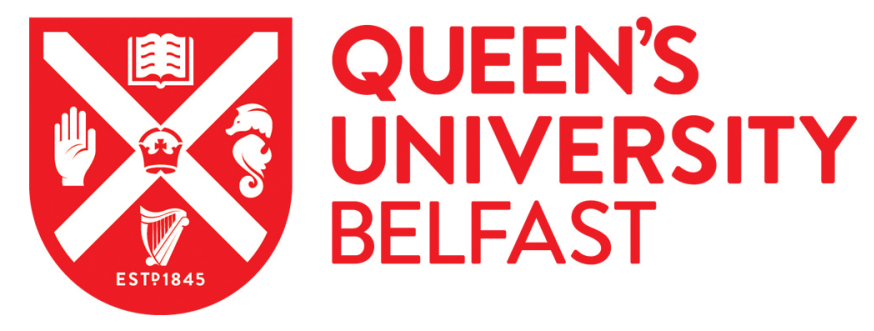

\title{
Replacing the Metal Electrodes in Triboelectric Nanogenerators: High- Performance Laser-Induced Graphene Electrodes
}

Zhao, P., Bhattacharya, G., Fishlock, S. J., Guy, J. G. M., Kumar, A., Tsonos, C., Yu, Z., Raj, S., McLaughlin, J. A., Luo, J., \& Soin, N. (2020). Replacing the Metal Electrodes in Triboelectric Nanogenerators: HighPerformance Laser-Induced Graphene Electrodes. Nano Energy, 75, [104958].

https://doi.org/10.1016/j.nanoen.2020.104958

Published in:

Nano Energy

Document Version:

Peer reviewed version

Queen's University Belfast - Research Portal:

Link to publication record in Queen's University Belfast Research Portal

Publisher rights

(c) 2020 Elsevier Ltd.

This manuscript is distributed under a Creative Commons Attribution-NonCommercial-NoDerivs License

(https://creativecommons.org/licenses/by-nc-nd/4.0/), which permits distribution and reproduction for non-commercial purposes, provided the author and source are cited.

\section{General rights}

Copyright for the publications made accessible via the Queen's University Belfast Research Portal is retained by the author(s) and / or other copyright owners and it is a condition of accessing these publications that users recognise and abide by the legal requirements associated with these rights.

Take down policy

The Research Portal is Queen's institutional repository that provides access to Queen's research output. Every effort has been made to ensure that content in the Research Portal does not infringe any person's rights, or applicable UK laws. If you discover content in the Research Portal that you believe breaches copyright or violates any law, please contact openaccess@qub.ac.uk. 


\section{Replacing the Metal Electrodes in Triboelectric Nanogenerators: High-Performance Laser-Induced Graphene \\ Electrodes}

Pengfei Zhao, ${ }^{1, \$}$ Gourav Bhattacharya, ${ }^{2, \$}$ Sam J. Fishlock,, 2, Joseph G. M. Guy, ${ }^{3}$ Amit Kumar, ${ }^{3}$ Christos Tsonos, ${ }^{4}$ Zidong Yu, ${ }^{1}$ Shasidran Raj, ${ }^{2}$ James A. McLaughlin, ${ }^{2}$ Jikui Luo ${ }^{5}$ and Navneet Soin $^{2 *}$

${ }^{1}$ Institute for Materials Research and Innovation (IMRI), School of Engineering, University of Bolton, Bolton BL3 5AB, United Kingdom

${ }^{2}$ School of Engineering, Ulster University, Newtownabbey, Belfast BT37 0QB, Northern Ireland, United Kingdom

${ }^{3}$ School of Mathematics and Physics, Queen's University Belfast, University Road, Belfast BT7 1NN, Northern Ireland, United Kingdom

${ }^{4}$ Department of Physics, University of Thessaly, $3^{\text {rd }}$ Old National Road, Lamia-Athens, 35100 Lamia, Greece

${ }^{5}$ College of Information Science and Electronic Engineering, Zhejiang University, 38 Zheda Road, Hangzhou 310027, P. R. China

\section{$\$$ Equal Contribution}

*Corresponding author email: n.soin@ulster.ac.uk 


\begin{abstract}
While significant work is being carried out to develop materials for enabling high surface charge density triboelectric nanogenerators (TENG), little attention has been paid to the role of electrodes responsible for charge collection. This work reports on the facile synthesis and use of high crystallinity, $\mathrm{sp}^{2}$-hybridised laser-induced graphene (LIG) as a high-efficiency electrode for triboelectric nanogenerators (TENGs). Using a conventional $10.6 \mu \mathrm{m} \mathrm{CO}$ laser, the controlled direct photothermal conversion of dielectric, tribo-negative polyimide (PI) and tribo-positive cellulosic paper into corresponding PI-LIG and paper-LIG, respectively, facilitates significantly higher electrical output as compared to the commonly utilised adhesive aluminium electrodes. The LIG based paper-PI TENGs showed significantly higher electrical output characteristics with a peak-to-peak voltage of up to $\sim 625 \mathrm{~V}$, a current density of $\sim 20$ $\mathrm{mA} \cdot \mathrm{m}^{-2}$ and a transferred charge density of $\sim 138 \mu \mathrm{C} \cdot \mathrm{m}^{-2}$ with a maximum power output of $\sim 2.25 \mathrm{~W} . \mathrm{m}^{-2}$, respectively while the corresponding values for the conventional Al-tape electrode based paper-PI TENGs were $\mathrm{V}_{\mathrm{p}-\mathrm{p}} 400 \mathrm{~V}, \sim 10 \mathrm{~mA} . \mathrm{m}^{-2}, \sim 85 \mu \mathrm{C} . \mathrm{m}^{-2}$ and $0.9 \mathrm{~W} . \mathrm{m}^{-2}$, respectively. The mechanically robust LIG electrodes show excellent stability with $<5.0 \%$ variation in output over 12,000 contact cycles. Using Kelvin probe force microscopy (KPFM) measurements, we have measured differences in not only the average surface potentials of the triboelectric surfaces $(-0.26 \mathrm{~V}$ for pristine PI $v s .+0.34 \mathrm{~V}$ for paper, which drive the TENG electrical output) but also for the LIG's synthesised from them (-0.08 V for PI-LIG $v$ s. +0.26 $\mathrm{V}$ for paper-LIG), suggestive of role of initial surface chemistry in the formation of LIGs. The enhanced ( $\sim 150 \%$ ) power density for LIG based TENGs is ascribed to the lowering of the charge transfer barrier height via the alignment of Fermi levels and resulting higher surface charge on the dielectric surface and the significantly ( 6 orders) lower interfacial contact impedance of LIG as compared to adhesive aluminium electrodes. Thus, via the removal of the additional interface between the triboelectric surface and electrode, high performance mwetalfree TENGs with excellent prospects for enabling energy harvesting applications can be realised.
\end{abstract}

Keywords: Laser-induced graphene; triboelectric nanogenerators; surface potential; energy harvesting; KPFM 


\section{Introduction}

Portable, wearable- electronic devices and technologies are fast becoming an indispensable part of our daily lives. These technologies are largely driven by the need of ubiquitous connectivity - known as IoT (Internet of Things), and is enabled by devices performing functions of health monitoring, environmental sensing, data sharing/processing by integrating multiple technologies, including sensors, real-time analytics, machine learning and embedded systems etc., for realising human-to-human or human-to-network interaction [1,2]. However, the limitations of the battery capacity have forced both the device and product designers to trade-off functionality and endurance for product size. The past few years have seen rapid development in the field of energy harvesting, especially triboelectric effect based nanogenerators which show the potential of enabling self-powered systems [3,4]. As a type of mechanical energy harvester, the simplest of triboelectric nanogenerators (TENG) can be assembled using commodity polymer materials and simple fabrication techniques. As compared to the other energy harvesters, TENGs have been shown to work more efficiently at low-frequencies $(1-10 \mathrm{~Hz})$ and at a relatively higher energy conversion efficiency of up to $85 \%$ [5]. As a result, since their invention in 2012, TENGs based on a combination of contact electrification and electrostatic induction effects are now regarded as one of the most effective means to harvest mechanical energy [4-7]. For the commonly reported vertical contactseparation mode TENG, two layers of tribo-charge with opposite polarity are formed on the interface via the contact of two dielectric materials with opposite triboelectric tendencies. The subsequent vertical periodic change of the distance between two materials causes a varying electric field which induces a periodic electric potential on the back-metal electrodes which usually comprise of a simple metal tape with acrylic based adhesive [8].

While the interfacial dielectric materials which directly participate in the tribo-charge generation (contact electrification) process are considered to be the most important component of TENGs, the material properties of the back electrodes are equally important affecting the output behaviour of the TENG. Till date, many conductive materials have been applied as the back electrodes, including gold [9,10], aluminium [11,12], copper [13] and carbon [14] etc. For designing TENG systems which can harvest energy from non-conformal surfaces, metal thin films show poor behaviour due to their unsuitable elastic behaviour (high Young's modulus) as compared to the triboelectric polymer surfaces. Moreover, the coating or adhesive process can affect the durability and life-time of the devices owing to the poor bonding of the metal electrode to the soft polymer contact layers [15]. These issues are fundamentally applicable to 
nearly all metallic thin films and maybe difficult to overcome [16]. Invariably, the poorly adhering electrodes result in a reduced electrical output as the charge collectors are not able to fully capture the generated charge [16]. As such, liquid metal (room temperature liquid) e.g. Galinstan based electrodes owing to their conductive, fluidic and highly conformal nature have been explored as charge collecting electrodes in TENGs [17]. However, when exposed to air, Galinstan oxidises readily, is difficult to handle and has only been shown to work in singleelectrode TENGs, which fundamentally has much lower electrical output than the verticalcontact mode TENGs [17]. Amongst all the reported electrodes, carbon-based electrodes are considered as an ideal option for realising effective electrical properties with high flexibility and examples of TENGs utilising multi-walled carbon nanotubes mesh [16] and graphene [18] have been reported. In a recent work, we have shown the effect of conductive carbon tape electrodes with coarse surface and high electronic affinity as a way to boost the electrical performance of TENGs, which was ascribed to a high density of pores between the triboelectric polydimethylsiloxane (PDMS) layer and the underlying carbon electrode [14]. As such the direct synthesis of microstructured conductive carbon ( $\mathrm{sp}^{2}$-carbon) on the polymeric contact layer to create the back electrode is an ideal option for realising high flexibility and a thorough, intimate electrical connection of the layers.

In 1990, Schumann et al. reported that $\mathrm{KrF}$ ultraviolet laser irradiation could convert polyimide (PI/Kapton) and polybenzimidazole into glassy carbon with permanently enhanced electrical conductivity [19]. In 2014, Lin et al. too demonstrated successful photothermal conversion of PI containing $\mathrm{sp}^{3}$-carbon atoms into three-dimensional networks which consists of $\mathrm{sp}^{2}$-carbon atoms and exhibited high electrical conductivity and flexibility. The films synthesised through this route is called as laser-induced graphene (LIG) [20]. Subsequently, Chyan et al. demonstrated that a wide range of carbohydrate-containing substrates (i.e. cellulose and lignin) could also be converted into LIG through a multiple pulsed laser scribing technique without losing the conductivity and the processing efficiency [21]. Recently, Stanford et al. realised a TENG based on the LIG electrodes directly formed on the surface of tribo-materials (PI, cork and PDMS), as an alternative to costly metal electrodes using vacuum deposition techniques [22] and time-consuming chemical vapour growth techniques [23]. However, the output power density from these dielectric-dielectric LIG TENGs was $\sim 0.76 \mathrm{~W} \cdot \mathrm{m}^{-2}$ only [22]. Similarly, Luo et al. have reported on the integration of micro-supercapacitors with TENGs, with LIG electrodes to realise a flexible self-charging power unit. However, their system provided a poor electrical performance with a maximum power output of only $0.8 \mathrm{~W} \cdot \mathrm{m}^{-2}$, owing to poor 
matching of the triboelectric surfaces [24].

Herein, we report on the use of laser induced graphene (LIG) synthesised directly on tribonegative polyimide (PI), and tribo-positive cellulose-based paper surfaces to fabricate highperformance TENGs. The highly crystalline $\mathrm{sp}^{2}$ LIG electrodes are flexible, robust, and are able to provide much higher outputs owing to the much more intimate contact with the triboelectric surface. The fabricated TENG shows a peak-to-peak voltage of up to $\sim 625 \mathrm{~V}$, a shortcircuit current density of $\sim 25 \mathrm{~mA} \cdot \mathrm{m}^{-2}$ (corresponding a charge density of $\sim 138 \mu \mathrm{C} . \mathrm{m}^{-2}$ ), and energy pulse of $\sim 11.8 \mu \mathrm{J}$.cycle ${ }^{-1}$ with an instantaneous power density of $2.25 \mathrm{~W} . \mathrm{m}^{-2}$, at a matched $100 \mathrm{M} \Omega$ load, respectively. All these values are significantly higher than the values for conventional aluminium electrode-based PI/paper TENGs. The LIG electrodes are mechanically stable and the developed TENG shows excellent stability with less than $\pm 7 \%$ variation in its electrical output over 12,000 contact cycles. In-depth characterization of the LIG electrodes show clearly the role of initial surface chemistry in the formation of subsequent LIGs wherein an average surface potential values of $-0.08 \mathrm{~V}$ for PI-LIG ( $v s .-0.26 \mathrm{~V}$ for pristine PI) and $+0.26 \mathrm{~V}$ for paper-LIG ( $v s .+0.34 \mathrm{~V}$ for paper) was observed, which then is reflected in the work function differences and the subsequent variation of the barrier. Furthermore, using dielectric relaxation spectroscopy, we have studied the interface between the dielectric and electrode to gain insight into the reasons for the enhanced performance of the LIG electrodes.

\section{Experimental}

\subsection{Materials and characterization}

Commercially obtained Polyimide (PI, 125- $\mu$ m thick PI, Kaneka, Apical NP, USA) and Whatman filter paper No. 1, were lased using a $10.6 \mu \mathrm{m}$ wavelength $\mathrm{CO}_{2}$ laser cutter (Universal Laser 230 VLS) under ambient conditions (Fig. 1 (a, b)). For the PI-LIG samples, the laser power was kept at $8.1 \mathrm{~W}\left(32.5 \%\right.$ of $25 \mathrm{~W}$ laser rating) with a scan speed of $570 \mathrm{~mm} \cdot \mathrm{s}^{-1}$. The PI-LIG electrode was designed using AutoCAD (Autodesk, 2017 USA) software and patterned as a $25 \times 40 \mathrm{~mm}^{2}$ block in raster mode at 1000 PPI ( $25.4 \mu \mathrm{m}$ separations between pulses). To produce paper-LIG, fire-retardant pre-treatment is necessary to avoid its decomposition to volatile compounds during the laser treatment [21]. For this, the paper substrate was treated using a commercial flame retardant (Firechief, UK). The lasing procedure was similar to that used for PI-LIG, except we lased the surface twice to obtain a high-quality paper-LIG electrode [21]. During the initial lasing run, the paper substrate was $0.8 \mathrm{~mm}$ out of focus (i.e. the laser focal point was $0.8 \mathrm{~mm}$ above the paper surface). Subsequently, the surface was laser-treated 
by in-focus laser. For both laser-runs on paper-LIG, the power was $1.5 \mathrm{~W}$ with the scan speed of $150 \mathrm{~mm} \mathrm{~s}^{-1}$.

A Hitachi SU5000 field emission scanning electron microscope (SEM) was used to study the surface morphology and cross-section of the LIG layer on PI and paper substrates. X-ray photoelectron spectroscopy (XPS) was performed on a Thermo Fisher Scientific NEXSA spectrometer using a micro-focused monochromatic Al X-ray source (19.2 W) over an area of approximately 100 microns. Data were recorded at pass energies of $150 \mathrm{eV}$ for survey scans and $40 \mathrm{eV}$ for high-resolution scans with $1 \mathrm{eV}$ and $0.1 \mathrm{eV}$ step sizes, respectively. Charge neutralization of the sample was achieved using a combination of both $\mathrm{Ar}^{+}$ions and low energy electrons. The Raman spectra were recorded using a Renishaw Raman spectrometer (inVia) using a $532 \mathrm{~nm}$ Laser source, using nominal power of $25 \mathrm{~mW}$ for $60 \mathrm{~s}$ at $50 \times$ magnification. Nanoscale surface potential mapping was undertaken using an Asylum Research MFP-Infinity atomic force microscope in the KPFM mode, which allows measurement of true surface potential through operation in a two-pass mode. For KPFM measurements, Pt-coated Si tips (Nanosensors PPP-EFM) with stiffness constant of $2.8 \mathrm{~N} . \mathrm{m}^{-1}$ were used.

\subsection{Preparation and electrical characterization of TENGs}

To prepare the conventional TENG control samples comprising of PI and paper substrates, conductive aluminium tapes ( $50 \mu \mathrm{m}$ thick) as electrodes were attached to two separate glass slides $(2 \mathrm{~cm} \times 2 \mathrm{~cm} \times 0.1 \mathrm{~cm})$. The LIG electrode-based TENG was produced by directly attaching the PI- or paper-LIG films to two glass substrates, with the LIG surfaces in contact with the glass while the PI- and paper- surfaces opposite to each other, as the tribo-negative and positive layers, respectively (see Fig. 1(h, i)).

A computer-programmable dynamic linear motor system (Linmot) combined with a force sensor (JHBM-H1) was used to simulate constant periodic mechanical input of the TENGs by controlling the contact force $(10-50 \mathrm{~N})$, frequency $(1-9 \mathrm{~Hz})$ at a fixed spacer distance $(5 \mathrm{~mm})$ of the two tribo-contact layers. An oscilloscope (Tektronix MDO3022 connected via a $100 \mathrm{M} \Omega$ input impedance high accuracy, high voltage passive probe) and a picoammeter (Keysight B2981A) were connected in parallel or serial, respectively, to measure the output voltage and the short-circuit current data for deriving the output current density and charge density, respectively. For all the TENG electrical measurements on picometer and oscilloscope, the electrodes corresponding to the paper sides were connected to the positive lead, while the PI side electrodes were grounded. 


\section{Results and discussion}

\subsection{Material characterization}

After the lasing process, a clear visual difference between the lased and pristine surfaces could be observed corresponding to the laser-induced pyrolysis/carbonisation. For both paper and PI substrates, it was observed that the optimal laser irradiation led to the formation of carbonbased material protruding from the starting substrate (Fig. 1(c, d)). In fact, the surface morphology of both the PI-LIG and paper-LIG clearly showed a loosely packed porous graphitic microstructure (Fig. 1(c, d)), similar to what has been reported by various groups including ours [21, 22, 25]. The uniform cross-sectional thickness of the PI substrate allowed clear visualisation of the PI-LIG and its interface with the pristine PI (Fig. 1(c)). Based on our experimental conditions, it was observed that $\sim 30 \mu \mathrm{m}$ of the PI substrate was ablated and converted into LIG while the opposite face of the PI substrate remained intact with no visible damage owing to the limited laser penetration depth and thermal properties of Kapton [26]. In the case of the paper substrate, owing to its fibrous nature (see Fig. S1, supporting information, for SEM image of pristine paper surface), the cross-sectional and interfacial measurements were difficult (Fig. 1(d)); however, the morphology of both the paper-LIG and PI-LIG appeared similar (Fig. 1(e, f)). For the process of the direct laser writing on PI substrates, Luo et al. have reported that the porous nature of the LIG films arises due to the "bombing" effect which leads to the release of $\mathrm{CO}_{2}, \mathrm{H}_{2} \mathrm{O}, \mathrm{H}_{2}$ and $\mathrm{CO}$ and other pyrolysis gases which also leads to an enhanced height of the synthesised LIG layer [27]. This hypothesis is underpinned by the earlier studies of Inagaki et al., wherein a two-step process of carbonisation of polyimides was observed [28]. The first-step occurring between $500-650{ }^{\circ} \mathrm{C}$ proceeds via the breakage of the carbonyl groups in the imide part which leads to an abrupt release of $\mathrm{CO}$ and $\mathrm{CO}_{2}$, while the second-step (occurring in the range $800-1000{ }^{\circ} \mathrm{C}$ ), leads to the release of $\mathrm{H}_{2}, \mathrm{O}_{2}$ and $\mathrm{N}_{2}$ accompanied by graphitisation [28, 29]. This pyrolysis/carbonisation process proceeds at a very fast rate owing to the rapid laser heating which is a characteristic of the laser-writing process [30]. It should, however, be noted that for both the cases, the unexposed side of the PI and paper substrates was not affected by the laser irradiation and could, therefore, be used directly as the triboelectric contact surface.

The Raman spectroscopy of both PI-LIG and paper-LIG samples was carried out to ascertain the nature and quality of the graphitic films. The presence of defective graphitic carbon was confirmed with a broad D band at $\sim 1350 \mathrm{~cm}^{-1}$, G band at $\sim 1580 \mathrm{~cm}^{-1}$ and the $2 \mathrm{D}$ peak near 
$2700 \mathrm{~cm}^{-1}$ for both the samples albeit with varying full width at half maxima (FWHM) values (see Fig. 1(g)) [31]. A simple comparison of the PI and paper-derived LIG shows that the $\mathrm{I}_{\mathrm{D}} / \mathrm{I}_{\mathrm{G}}$ ratio for the PI-LIG was marginally higher (0.82) as compared to the paper-LIG (0.79) and can be attributed to the differences in the chemical bonding. The thermally stable polyimides contain aromatic backbone structures composed primarily of fused rings and rings directly linked by carbon-carbon bonds whereas the paper contains largely aliphatic carbon in the form of cellulose (see XPS discussion) [32]. It has been shown previously that aliphatic carbons are more easily decomposed and produce more defects on laser irradiation, and hence the observed differences in the $\mathrm{I}_{\mathrm{D}} / \mathrm{I}_{\mathrm{G}}$ ratio can be easily understood $[32,33]$. In the second-order Raman spectra, the FWHM of the fitted 2D peak for PI-LIG is slightly higher $\left(83 \mathrm{~cm}^{-1}\right)$ than that of the paper-LIG $\left(72 \mathrm{~cm}^{-1}\right)$ which is typical for $3 \mathrm{D}$ graphitic films consisting of randomly stacked graphene layers along the dominant c-axis [20]. Concurrently, the $\mathrm{I}_{2 \mathrm{D}} / \mathrm{I}_{\mathrm{G}}$ value of approx. 0.68 for PI-LIG and 0.55 for paper-LIG, respectively, further confirms the excellent graphitisation $[20,32-34]$.

In order to understand the compositional changes occurring upon the laser irradiation, XPS analysis of the pristine PI, paper and their corresponding derived LIGs has been carried out (Fig. 2). For the pristine PI film, the surface chemical composition was measured to be $\mathrm{C}$ (78.05 at.\%), O (17.30 at.\%) and $\mathrm{N}(4.65 \mathrm{at} \%)$ which matches well with the theoretical and reported values $[34,35]$. The high-resolution C1s spectra for the pristine PI (Fig. 2(a), left panel) was deconvoluted into four main functional groups: carbon in aromatic rings $(\mathrm{C}-\mathrm{C})$ not attached to the imide ring $(284.6 \mathrm{eV})$, carbon atoms bonded to nitrogen $(\mathrm{C}-\mathrm{N})$ at $285.6 \mathrm{eV}$, carbon atoms single-bonded to oxygen $(\mathrm{C}-\mathrm{O})$ at $286.4 \mathrm{eV}$ and carbonyl groups $(\mathrm{C}=\mathrm{O})$ present at $288.7 \mathrm{eV}$, respectively. For the PI core-level O 1s spectra (Fig. 2(b)), two components corresponding to the $\mathrm{C}=\mathrm{O}$ and $\mathrm{C}-\mathrm{O}$ bonding were observed at $\sim 532.1$ and $533.4 \mathrm{eV}$, respectively [36]. A symmetrical peak arising from the $\mathrm{C}-\mathrm{N}$ bonding at $\sim 400.6 \mathrm{eV}$ was observed for the $\mathrm{N} 1$ s corelevel region (inset Fig. 2(b)) [35, 36]. After the laser ablation process to form PI-LIG, significant changes in the core-level spectra were observed with the components at $285.6 \mathrm{eV}$ (C-N), $286.4 \mathrm{eV}(\mathrm{C}-\mathrm{O})$ and $288.7 \mathrm{eV}(\mathrm{C}=\mathrm{O})$ reduced significantly while components at $\sim 285.0$ $\mathrm{eV}$, corresponding to $\mathrm{C}-\mathrm{C}$ and $\mathrm{C}=\mathrm{C}$ bonding increasing dramatically (Fig. 2(a), right panel and Fig. 2(c)). Correspondingly, the surface composition of the PI-LIG changed dramatically wherein most of the surface oxygen/nitrogen was removed (C (92.57 at\%), O (6.53 at\%) and $\mathrm{N}(0.90$ at $\%)$ ) with the corresponding $\mathrm{C} / \mathrm{O}$ and $\mathrm{C} / \mathrm{N}$ atomic ratios changing from 4.5:1 and 16.8:1 (for pristine PI) to 14.2:1 and 102:1, respectively (inset Fig. 2(a)). These compositional 
values support the earlier discussion and confirm that the $\mathrm{CO}_{2}$ laser irradiation of PI films releases both oxygen and nitrogen (amongst other pyrolysis gases) as volatile species and the residual films are largely $\mathrm{sp}^{2}$ carbons only [28, 29, 35, 36].

Similarly for the paper substrate, while the initial surface chemistry is dominated by C $(55.02$ at.\%) and $\mathrm{O}$ (41.80 at.\%), trace values of S (1.68 at.\%) and $\mathrm{N}$ (1.50 at.\%) were also detected (Fig. 2(d), left panel) [37]. After the $\mathrm{CO}_{2}$ lasing process, the paper-LIG surface composition was predominantly C (72.79 at.\%) and O (20.02 at.\%) with some trace quantities of B (3.79 at.\%), S (2.53 at.\%) and N (0.88 at.\%), see Fig. 2(d) right panel and Fig. 2(f). The presence of Boron is ascribed to the fire-retardant spray, which was sprayed onto the paper substrates [21, 33]. As discussed earlier, for cellulosic materials, fire-retardant pre-treatment is necessary to avoid their decomposition to volatile compounds during the laser treatment [21, 33]. The significant difference in the residual oxygen content of the paper-LIG can be ascribed to the effects of the physio-chemical structure (randomly laid fibrous (cellulose) and non-fibrous (fillers) materials) of the paper as well as the presence of the fire-retardant in the matrix which reduces ablation. As cellulose and hemicellulose are prone to burning at relatively low temperatures, the borylation of the paper substrate helps catalyse dehydration and oxygenelimination reactions of cellulose, resulting in an amorphous char high in aromatic content which is then converted to paper-LIG during the lasing process during which the corresponding $\mathrm{C} / \mathrm{O}$ and $\mathrm{C} / \mathrm{N}$ atomic ratios changing from 1.32:1 and 36.68:1 (for pristine paper) to 3.63:1 and 82.71:1, respectively [21, 33, 37] (see inset Fig. 2(d)). It should be noted that these results are very similar to those reported by Lee et al. in their recent work on laser-induced graphitisation of cellulose substrates [37]. This significant difference in the surface chemistry of the LIG films is also reflected in their respective surface contact potentials and is discussed in the later section.

\subsection{Electrical characterization of TENGs}

To assess the suitability of the LIG as charge collection electrode, we have undertaken comparative studies of the following TENG configurations: pristine PI (tribo-negative) vs. pristine paper (tribo-positive) with Al back electrodes (control) and PI vs. paper with LIG electrodes (see Fig. 1(g, h)). The measured electrical output performance of the PI/paper based TENG with $\mathrm{Al}$ and LIG electrodes is shown in Fig. 3. It is understood that under compressive forces when the triboelectric surface such as paper/PI surfaces are in contact, equal and opposite triboelectric charges, i.e. negative charges on the PI and positive charges on the paper are generated $[11,12,38]$. Upon the removal of the force, the presence of these opposite 
charges on the surfaces leads to the formation of the potential between the two metal electrodes, which can drive the electrons through an external circuit until the potential difference is neutralized. As can be observed clearly, within the range of the measured contact forces, both the Al (Fig. 3(a)) and LIG (Fig. 3(c)) electrode based TENGs exhibited a linear relationship between the voltage output and the contact force. While the peak-to-peak voltage, $\mathrm{V}_{\mathrm{p}-\mathrm{p}}$, of the Al electrode-based TENG (Fig. 3(a)), showed a relatively small increase in the voltage outputs ranging from $\sim 260 \mathrm{~V}_{\mathrm{p}-\mathrm{p}}$ (at $10 \mathrm{~N}$ ) to $\sim 400 \mathrm{~V}_{\mathrm{p}-\mathrm{p}}$ (at $50 \mathrm{~N}$ ), the LIG (Fig. 3(c)) based TENG showed consistently higher values ranging from $\sim 360 \mathrm{~V}_{\mathrm{p}-\mathrm{p}}$ at the minimum contact force of 10 $\mathrm{N}$ to $\sim 635 \mathrm{~V}_{\mathrm{p}-\mathrm{p}}$ (at $50 \mathrm{~N}$ ), respectively. Correspondingly, the Al electrode-based TENG (Fig. 3(b)) was only able to provide a maximum current density and transferred charge density values (at $50 \mathrm{~N}$ ) of $\sim 10 \mathrm{~mA} . \mathrm{m}^{-2}$ and $\sim 85 \mu \mathrm{C} . \mathrm{m}^{-2}$, respectively. Comparatively, the LIG based TENG showed higher short-circuit current density and effective charge density values of $\sim 20 \mathrm{~mA} . \mathrm{m}-$ 2 and $138 \mu \mathrm{C} . \mathrm{m}^{-2}$, respectively (Fig. 3(d)). According to literature and indeed our previous reports, the impact force and impact frequency are the two most important operating parameters which control the electrical output of the TENGs [11, 12, 38-40]. For both the LIG and Al based PI-paper TENGs, an increase in the charge density values and the voltage/current peak values across the force range was observed which arises from the growth of the transferred charge amount induced by higher surface tribo-charge density. The increase of the generated tribo-charge density itself with increasing force can be attributed to the combined effect of the mechanical stress-induced higher contact area, and the mechanochemical phenomenon as the localised stress which is further magnified by the roughness of the paper substrate $[11,12,38$ 40]. As the LIG based TENGs were producing significantly higher electrical output, further measurements of the frequency dependence were limited to these LIG PI-paper TENGs only.

As shown in Fig. 3(e), by varying the contact frequency $(1-9 \mathrm{~Hz})$, the magnitude of the LIG based PI-paper TENG output voltage peak was dramatically enhanced from $\sim 300 \mathrm{~V}_{\mathrm{p}-\mathrm{p}}$ to $\sim 625$ $\mathrm{V}_{\mathrm{p}-\mathrm{p}}$; meanwhile, the peak short-circuit charge density increased from $\sim 3.6 \mathrm{~mA} . \mathrm{m}^{-2}$ to $\sim 22.0$ $\mathrm{mA} \cdot \mathrm{m}^{-2}$. However, contrary to the phenomenon observed from force-output results, for the energy harvesting purpose, the most crucial output parameters, transferred charge density gradually decayed from $\sim 207 \mu \mathrm{C} . \mathrm{m}^{-2}$ to $\sim 113 \mu \mathrm{C} . \mathrm{m}^{-2}$ along with the increase of the operating frequencies, which contradicted to the growth of the magnitude of the output voltage and current. This behaviour is different from the normally observed behaviour in TENGs wherein the charge density and current density have a direct relationship [38-40]. It is understood that for the contact-mode TENGs, the output current is influenced by the interaction between the 
triboelectric layers, i.e. the instantaneous current during the releasing process is strongly affected by the separation speed, see Eq. 1 below [38-40, 41]. Under short-circuit conditions, the transferred charge for the contact-separation mode TENGs is represented by the following relationship:

$$
Q_{s c}=\frac{S \sigma x(t)}{d_{0}+x(t)}
$$

Where $S$ is the plane contact area, $\sigma$ is the surface electrification charge density, $x(t)$ is the realtime distance between two contact layers, and $d_{0}$ is the effective thickness represented by:

$$
d_{0}=\frac{d_{1}}{\varepsilon_{1}}+\frac{d_{2}}{\varepsilon_{2}}
$$

Where $\varepsilon_{1}$ and $\varepsilon_{2}$ represent the relative dielectric constants of the two contact triboelectric contact layers of thicknesses $d_{1}$ and $d_{2}$, respectively [41].

The singular waveform of the current outputs obtained in different operation frequencies is shown in Fig. S2 (supplementary information) where it can be seen that the lower operating frequency allows longer charge transfer time between the two electrodes, therefore higher amount of charge (derived by integrating the area under the singular cycle pulse of the current density) can be expected. As it can be clearly observed, even though the peak current density is increasing, the current pulse width is getting narrower with the increasing frequency owing to the significantly shorter contact time between the two triboelectric interfaces [39]. The high operation frequency results in sharper and higher output peaks, which can be useful in the applications of the requirements for high voltage/signal amplitude, i.e. as a power supply of an electrospinning system, plasma stimulation or excitation signal of a RF (Radiofrequency) wireless communication system [42]. Moreover, unlike the common TENG systems such as PA-6/PTFE [11, 12], PEO/PTFE or PDMS/PEO [40] where the effective stiction between the two "soft-polymers" is high which leads to a relatively constant release time between the triboelectric surfaces, the relative lack of stiction between the PI and paper surfaces also contributes to the observed variation in the growth trend of current and transferred charge density.

The output power of both the Al and LIG based PI-paper TENGs was tested by connecting various electrical loads in series at a constant operating condition of $50 \mathrm{~N}$ force, $5 \mathrm{~mm}$ spacer distance and $5 \mathrm{~Hz}$ working frequency. The maximum output power of the LIG or Al electrodebased TENGs at impedance matching condition was tuned by connecting various external load 
resistors in series. The accurate instantaneous peak power values can thus be measured by measuring the current across each resistor,

$$
\mathbf{P}=\mathbf{I}_{\text {peak }}{ }^{2} \mathbf{R}
$$

where $I_{\text {peak }}$ stands for the maximum current value measured current across the load resistance across each load resistors, $R$. It can be observed in Fig. 4(a) that the maximum output power of both $\mathrm{Al}$ and LIG based TENG devices was obtained at a load resistance of $\sim 100 \mathrm{M} \Omega$. At the impedance matching conditions, the LIG electrode-based TENG can output a power density of nearly $\sim 2.25$ W.m ${ }^{-2}$, which more than 2.5 times higher than the Al electrode-based one $(\sim 0.9$ $\mathrm{W} . \mathrm{m}^{-2}$ ). It should be noted that the reported power of the PI-paper LIG TENG is nearly 3 times higher than the value $\left(0.76 \mathrm{~W} . \mathrm{m}^{-2}\right)$ reported by Stanford et al. [22] and Luo et al. $\left(0.8 \mathrm{~W} . \mathrm{m}^{-2}\right)$ [24] for such LIG based dielectric-dielectric TENGs. As illustrated in the frequency-output characterisation section, it has been demonstrated that the magnitude of the instantaneous output peaks mainly depends on the duration of the charge transfer time instead of the amount of charge transferred. Therefore, for energy harvesting/conversion purpose, the energy $(E)$ derived from the area under the power curve of each operation cycle at the impedance matching condition is much more representative of the output electric energy characterization. This energy, $E$, is given by:

$$
E=\int_{n t}^{(n+1) t} I_{t}^{2} R_{l o a d} d t
$$

where $I$ denotes the measured current values across the impedance-matching load resistance, $n$ is the number of generation cycle, and $t$ is the time period of each energy generation cycle $(0.2$ $\mathrm{s}$ at $5 \mathrm{~Hz}$ working frequency) [39]. At the impedance matching condition (when $\mathrm{R}_{\mathrm{Load}}=100$ $\mathrm{M} \Omega$ ), the harvested energy was calculated to be $\sim 11.8 \mu \mathrm{J}_{\text {.cycle }}{ }^{-1}$ (Fig. 4(b)), which is twice the harvested energy from an $\mathrm{Al}$ electrode-based one $\left(\sim 5.5 \mu \mathrm{J}\right.$.cycle $\left.{ }^{-1}\right)$ and similar to the energy harvested from a PA6/electrospun-PTFE based TENG reported in our earlier work $(\sim 13.8 \mu \mathrm{J}$ cycle $\left.^{-1}\right)[12]$.

The output stability of the LIG based TENGs was further demonstrated by monitoring the output variation over a long-period operation (over 12,000 energy generation cycles at 50 N, 5 $\mathrm{Hz}$ conditions). As shown in Fig. 4(c, d), both the voltage and current outputs initially gradually dropped down by $\sim 5-7 \%$ in the initial $900 \mathrm{~s}$ operation time; however, no further attenuation was observed afterwards. The continuous stable output demonstrates not only the duration of the tribo-charge on the selected dielectric material surfaces but also the sufficient mechanical 
strength of the underlying LIG electrode layer. It is worth mentioning that all the previous electrical characterisations were measured within the stable region after a period of preoperation time. We have further utilised this metal-free PI-Paper LIG based TENG to power up an electronic calculator using fly-buck converter circuit we had developed in our earlier works (Fig. 4(e, f)) [40]. The fly-buck circuit (Fig. 4(e)) not only provides a higher efficiency as compared to a standard full-wave rectifier but also provides faster charging capability [40]. Owing to this capability of the circuit, a $2 \times 2 \mathrm{~cm}^{2}$ PI-paper LIG TENG working at a frequency of $3 \mathrm{~Hz}$, was able to drive a calculator, in real-time, whose coin cell batteries had been removed (See Supplementary video 1). Even after the TENG stopped working, the calculator was able to function for tens of seconds owing to the energy stored in the capacitor (See Supplementary video 2). Thus, the LIG based TENG devices can provide significant power densities suitable to power up electronic devices and at the same time are robust enough to sustain a long-term operation.

\subsection{Why do LIG electrode based TENGs perform better?}

In order to understand the output behaviour of the samples, we have investigated the material chemistry, dielectric relaxation mechanism and measured the average surface potential of the triboelectric surfaces as well as that of the LIG electrodes using KPFM. The KPFM measurements rely on the matching of tip bias $\mathrm{V}_{\mathrm{DC}}$ with the contact potential difference between the sample and the probe, $\mathrm{V}_{\mathrm{CPD}}$, by nullifying the vibration of the probe, which is initially driven by the electrostatic force, induced on the AFM probe. It should be noted that the mapped surface potential or the contact potential difference $\left(\mathrm{V}_{\mathrm{CPD}}\right)$ has contributions arising from not only the differences in the work functions but also from the presence of uncompensated charges, particularly for polymeric and non-metallic samples [39]. The average surface potential of pristine PI (Fig. 5(a, b)) was measured to be $-0.26 \mathrm{~V}$, while for the paper substrate (Fig. 5(e, f)), the corresponding distribution of the surface potential was much broader with a peak value at around $+0.34 \mathrm{~V}$. A higher surface potential implies that the electrons can be easily donated from the surface and the surface will gain a positive charge after contacting with the other materials [39]. Thus, as compared to the PI, the paper is triboelectrically positive, thus having a higher ability to donate electrons and explains the performance obtained for the paper/PI TENG. It should be noted that this difference between the surface potentials of the two dielectric surfaces is not as wide as for other classical triboelectric systems such as PTFE/PA-6 [12], PEO/PDMS [40] or more recently developed AFR/PTFE [39] and is subsequently reflected in the relatively lower output of the paper/PI TENG and demonstrates 
the need for judicious control of the materials for TENG devices. To further ascertain how the formation of LIG changes the surface potential of the electrodes and if that has a role in the ensuing performance of the LIG based TENGs, we have also measured the KPFM response of the paper-LIG and PI-LIG. For comparison, we have also measured the surface potential of standard Al tape electrodes ( 0.9 V, Fig. 5(i)) which matches well with the value expected due to the differences in the work function of the Pt tip $(5.2 \mathrm{eV})$ and that of $\mathrm{Al}(4.2 \mathrm{eV})$.

While it would be expected that the laser ablation of the surfaces should essentially remove the majority of polymer functional groups and erase the chemical history of the pristine surfaces, surprisingly, we observed a significant measurable difference between the surface potential of the LIG obtained on the paper and on the PI substrates. While the PI-LIG still showed a relatively negative surface potential of $-0.082 \mathrm{~V}$ (Fig. 5 (c, d)), the paper-LIG showed a value of $+0.26 \mathrm{~V}$ (Fig. $5(\mathrm{~g}, \mathrm{~h})$ ), suggesting a significant role of initial surface chemistry in the subsequent formation of LIGs and effects of residual surface chemistry. To understand this difference, we need to revisit the XPS measurements of the surface functional groups present on the LIG surfaces. As compared to the surface composition of PI-LIG ((C (92.57 at\%), O (6.53 at\%) and $\mathrm{N}(0.90 \mathrm{at} \%))$, the paper-LIG has much more significant amount of residual oxygen on its surface (C (72.79 at.\%) and O (20.02 at.\%)) and the presence of residual C-O and $\mathrm{C}=\mathrm{O}$ groups (see Fig. 2(d) right panel and Fig. 2(f)) provides a positive charge onto the surface which is thus reflected in its positive surface contact potential $[39,40]$. This agrees well with our earlier reports $[39,40]$ and that of Diaz et al. [43] where it was reported that surfaces with oxygen functional groups develop the highest positive charge next only to nitrogenated groups.

Now, it is understood that when two surfaces come into contact with each other, they will exchange charge as determined by their Fermi level $\left(E_{\mathrm{F}}\right)$ and work function $(\varphi)$ differences, where a material with a higher work function will act as an electronegative material and accept electrons from a lower work function electropositive material [41, 44, 45]. It is well established that for contact mode TENGs, a larger difference in the work function values of the two triboelectric contact surfaces drives a higher charge exchange [45]. As the charges get exchanged, they create an electrostatic potential difference which tends to pull them together, thus creating a contact potential at the point of contact that counteracts the work function difference [45]. However, in nearly all the studies, the initial charge transfer occurring during the formation of the intimate contact between the metal back electrode, e.g. $\mathrm{Al}$ and the dielectric surface is ignored. In this respect, recent work by $\mathrm{Xu}$ et al. has reported on the role of metal 
work function, $E_{\mathrm{F}}, V_{\mathrm{CPD}}$ and the resulting barrier height $(W)$ in controlling the tendency and direction of the contact electrification [46]. Using a classic model of dielectric and a metal, the "regulation" i.e. altering the barrier height upon contact between a dielectric and metal was discussed elegantly [46].

To gain a better understanding of the role of electrodes, we have carried out TENG electrical output measurements on identical triboelectric materials for various combinations of electrodes viz. PI-LIG/PI vs. PI/PI-LIG, Al/PI vs. PI/Al and Al/PI vs. PI/PI-LIG, by using the proposed model by Xu et al. [46]. The idea of using identical triboelectric materials was to exclude the electrical output arising from the contact electrification between the dielectrics of different work functions which can overshadow the electrode effects. Voltage and current output for various electrode combinations are shown in Fig. 6(a,b). While it is expected that for the same tribo-materials, a significant electrical output should not be observed owing to the similar nature of the chemical surfaces, we do observe a significant role of the electrode in these measurements. In the case of LIG electrodes (PI-LIG/PI vs. PI/PI-LIG), negligible electrical output was observed $\left(<8 \mathrm{~V}_{\mathrm{p}-\mathrm{p}}, 0.2 \mu \mathrm{A}\right)$ which can be ascribed to the near matching of the work functions of the PI $(5.5 \mathrm{eV})$ and PI-LIG $(5.4 \mathrm{eV})$, which prevented any significant charge transfer between the PI and PI-LIG (Fig. 6(c)). As a result, the neutral states, $E_{N}$, were formed in PI which should be near the $E_{\mathrm{F}}$ of PI-LIG (Fig. 6(c)) and no significant charge transfer occurs [46]. In the case of $\mathrm{Al}$ tape electrodes on PI, the work function differences between the dielectric and metal are much more significant (Al $4.2 \mathrm{eV}$, PI $5.5 \mathrm{eV}$ ), which would lead to an alignment of the Fermi levels where electrons would be transferred from Al to PI, endowing the PI surface with a negative charge and leading to the formation of $E_{\mathrm{N}}$ states in PI (Fig. 6(d)) [46]. Considering this TENG configuration, either of the two Al/PI surfaces could potentially have a slightly higher initial negative charge (owing to the local variations in surface chemistry, morphology etc.). Now, when these two surfaces meet each other, with the $E_{\mathrm{N}}$ being at similar levels, for the electron transfer to take place, only the barrier $W$ needs to be overcome (Fig. 6(e)). This coupled with the initial surface charge produced during the Fermi level alignment, leads to a higher electrical output than as compared to the PI-LIG/PI vs. PI/PI-LIG TENG [46]. In the case of PI-LIG/PI vs. PI/PI-LIG even though the barrier $W$ should be quite small, there was no significant initial charge to transfer across it and hence poor output was observed. Finally, in the case of Al/PI vs. PI/PI-LIG TENG, on the Al/PI side the initial contact between the electrode and the dielectric will lead to the production of a significant initial charge owing to the large differences in their work functions (PI surface in contact with Al will develop a 
negative charge). Whereas on the PI/PI-LIG side, the near matching of the work functions leads to the absence of any significant initial charge. In such a scenario, the $\mathrm{Al} / \mathrm{PI}$ side can be assumed to act as an overall charge provider (tribo-positive), while the PI/PI-LIG side would be an effective electron acceptor (tribo-negative) system with differences in their subsequent $E_{\mathrm{N}}$ states (Fig. 6(f)). The ensuing contact electrification comprising of such as system (Al/PI) with an initial charge and dielectric with no significant charge (PI/PI-LIG) leads to a lowering of the barrier $W$ by $\Delta E(W-\Delta E)$ which therefore improves the charge transfer and the overall electrical output of the system [46]. By lowering the barrier via direct synthesis of metal-free electrodes on triboelectric surfaces, significantly higher charge density can therefore be extracted from the TENGs.

It should be noted that in our earlier work, we have reported on the effect of electrode porosity on the TENG electrical output, wherein a device based on microporous carbon electrode showed significantly higher output than conventional adhesive-metal electrodes [14]. For the PI-LIG and paper-LIG electrodes, due to the observed significant micro-porosity, an enhancement in the electrical output is expected. We have further analysed the dielectric/electrode interface using dielectric relaxation spectroscopy in order to characterize the resistance arising from the interface. The dependence of the real part, $\varepsilon^{\prime}$, of the dielectric function $\varepsilon^{*}=\varepsilon^{\prime}-j \varepsilon^{\prime \prime}$, on the frequency $f$ is shown in Fig. 7(a) for Al-adhesive-Al (Al-ADH$\mathrm{Al}$ ), pristine PI and Al-Polyimide-LIG (Al-PI-LIG) samples. The $\varepsilon^{\prime}$ value of the pristine PI is almost constant across the frequency range and takes a value close to $\varepsilon^{\prime}=2.2$ [47] but does not show any relaxation events in this frequency range. For the configuration Al-ADH-Al, a highfrequency step is observed (Fig. 7(a)) which is due to the existence of dielectric relaxation, while for the intermediate frequencies, $\varepsilon^{\prime}$ is almost constant with a value close to $\varepsilon^{\prime}=3.4$. A slight increase of $\varepsilon^{\prime}$ value at the lower frequencies is observed due to electrodes effect, as the direct current (DC) conductivity dominates at this frequency range and is discussed below. In the Al-PI-LIG system, as shown in Fig. 7(a), a step in $\varepsilon^{\prime}$ values is present at lower frequencies which corresponds to a polarization mechanism. A high-frequency step is observed similar to the one observed for the Al-ADH-Al, which arises due to the dielectric relaxation of adhesive. The dependence of the alternating current (AC) conductivity $\sigma^{\prime}=\varepsilon_{o} \varepsilon^{\prime \prime} \omega$ (where $\omega=2 \pi f$, angular frequency) on frequency $f$ is shown in Fig. 7(b) for the Al-ADH-Al, Pristine PI and AlPI-LIG samples. A clear low-frequency plateau is observed in Al-ADH-Al which corresponds to a DC conductivity value of $\sigma_{\mathrm{dc}}=5.2 \times 10^{-13} \mathrm{~S} / \mathrm{cm}$. The DC conductivity is also given by the relation $\sigma_{d c}=\varepsilon_{o} \varepsilon^{\prime \prime} \omega_{M}$, where $\omega_{\mathrm{M}}$ corresponds to the peak frequency $f_{\mathrm{M}}$ of conductivity 
relaxation mechanism in electric modulus $M^{*}$ representation [48]. As shown in Fig. 7(c), which presents the data of the imaginary part $M^{\prime \prime}$ of electric modulus, $M^{*}=M^{\prime}+j M^{\prime \prime}$, the lower frequency relaxation of Al-ADH-Al takes a maximum value at frequency $f_{\mathrm{M}}=0.27 \mathrm{~Hz}$. So, according to the previous relation the $\mathrm{DC}$ conductivity of adhesive for the Al-ADH-Al sample is calculated to be $\sigma_{\mathrm{dc}}=5.1 \times 10^{-13} \mathrm{~S} / \mathrm{cm}$, a value almost identical to that extracted from the low frequency plateau. This fact indicates that the lower frequency mechanism (Fig. 7(c)) corresponds to the conductivity relaxation mechanism of adhesive of $\mathrm{Al}$ tape [49]. Based on the previous analysis, the low frequency peak in $M^{\prime \prime}$ of Al-PI-LIG at $7.5 \mathrm{~Hz}$ can therefore be related to the DC conductivity relaxation of adhesive of the Al tape. The existence of the previous relaxation also leads to a polarization mechanism in the total structure, which causes the lower frequencies step observed in $\varepsilon^{\prime}$ values (Fig. 7 (a)).

In order to carry out calculations on the adhesive interface characteristics of Al-PI-LIG, an equivalent circuit of a parallel $R$ - $C$ element was assumed which describes its dielectric response, where $C$ is the capacitance and $R$ is the resistance of the adhesive [50]. According to the previous model, the maximum value $M^{\prime \prime}{ }^{\prime}{ }_{\max }=0.018$, corresponding to the conductivity relaxation mechanism of the adhesive interface in Al-PI-LIG (Fig. 7(c)), is related to the capacitance $C$ via the relation $M_{\max }^{\prime \prime}=C_{o} / 2 C$, where $C_{\mathrm{o}}=16.8 \mathrm{pF}$ is the geometrical capacitance of the sample [51]. Based on the previous relationship, a capacitance value of $C=$ $467 \mathrm{pF}$ is calculated for the adhesive interface of Al-PI-LIG. On the other hand, the relaxation time, $\tau$, of the R-C equivalent circuit is equal to $\tau=R C$ where $\omega_{\mathrm{M}} \tau=1, \omega_{\mathrm{M}}=2 \pi \mathrm{f}_{\mathrm{M}}$ with $f_{\mathrm{M}}=$ $7.5 \mathrm{~Hz}$ the frequency corresponding to the maximum value of $M^{\prime \prime}$ of the conductivity relaxation mechanism. Taking into account the previous value of capacitance, $C=467 \mathrm{pF}$, a value of $R=$ 45.5 $\mathrm{M} \Omega$ is extracted and corresponds to the resistance of the adhesive interface in Al-PI-LIG structure.

It should be noted that the interfacial resistance for the PI-LIG interface is expected to be much lower i.e. more conductive than the Al-PI adhesive interface, because of the presence of LIG phase. In Figs. 7 (a-c) no relaxation event or contribution was observed in the frequency range $0.1 \mathrm{~Hz}-1 \mathrm{MHz}$ which could be ascribed to the PI-LIG interface. Therefore, it can be safely assumed that the possible relaxation event associated with the PI-LIG interface could therefore occur at a frequency $\mathrm{f}_{\mathrm{M}}$ (maximum value of $\mathrm{M}$ ') higher than $10 \mathrm{MHz}$. On the other hand, typical values characterizing the capacitance of PI-LIG interface, assuming it behaves as a surface layer, are in the range $0.01 \mathrm{nF}-1 \mathrm{nF}$ [52]. Thus, in order to estimate the upper limit of PI-LIG interface resistance value $R_{\max }$, the lower values of frequency $\mathrm{f}_{\mathrm{M}}=10 \mathrm{MHz}$ and 
capacitance $\mathrm{C}=1 \mathrm{nF}$ were used according to the equivalent circuit of the parallel $\mathrm{R}-\mathrm{C}$ elements. Then, taking into account the relation $\omega_{\mathrm{M}} \tau=1$ with $\tau=R C$ the maximum value of PI-LIG interface was found to be $R_{\max }=16 \Omega, 6$ orders of magnitude lower than this of Al-PI adhesive interface. Considering all the arguments provided in the previous sections, we attribute the greatly improved ( $150 \%$ ) power output of the TENG based on LIG electrodes to a multitude of mechanisms, including (i) the LIG electrode with a microporous structure; (ii) the alteration in Fermi levels caused by the contacting triboelectric surfaces and back electrodes leading to an effective alteration in the initial surface charge of the contacting surfaces, thereby increasing the amount of charge transferred and (iii) a significantly reduced interfacial resistance between the triboelectric material and contacting electrode acts to decrease the overall resistance of the structure and thereby increasing the power density. Therefore, by eliminating the resistive interface between the triboelectric surface and the metal back electrode, significantly higher charge density can therefore be extracted from the TENGs.

\section{Conclusions}

In summary, using a conventional $\mathrm{CO}_{2}$ laser we have synthesised crystalline $\mathrm{sp}^{2} \mathrm{LIG}$ electrodes directly on the surfaces of tribo-positive and tribo-negative materials to significantly enhance the power output of the metal-free TENGs by $150 \%$. The LIG electrode based paper-PI TENGs exhibit a peak-to-peak voltage of up to $\sim 625 \mathrm{~V}$, a current density of $\sim 20 \mathrm{~mA} \cdot \mathrm{m}^{-2}$ and a transferred charge density of $\sim 138 \mu \mathrm{C} . \mathrm{m}^{-2}$ with a maximum power output of $\sim 2.25 \mathrm{~W} . \mathrm{m}^{-2}$, as compared to $400 \mathrm{~V}, \sim 10 \mathrm{~mA} . \mathrm{m}^{-2}, \sim 85 \mu \mathrm{C} . \mathrm{m}^{-2}$ and $0.9 \mathrm{~W} . \mathrm{m}^{-2}$ for conventional Al electrode based paper-PI TENGs, respectively. The enhanced performance of LIG electrode based paper-PI TENG is ascribed to a combination of formation of intimate electrical contact between the electrode and triboelectric surface thereby excluding any additional high resistance interface (such as adhesive when using aluminium tapes) and the lowering of potential barrier improving the charge transfer. Additionally, we have observed the effects of initial surface chemistry on the surface potential of the subsequent LIG electrodes wherein the tribo-positive surface $(+0.34$ $\mathrm{V}$ for pristine paper) maintained an average positive surface potential $(+0.26 \mathrm{~V})$, while the tribo-negative surface $(-0.26 \mathrm{~V}$ for pristine $\mathrm{PI})$ maintained its negative surface potential (-0.08 V) even after the $\mathrm{CO}_{2}$ lasing operation. The LIG electrodes are robust and mechanically stable, displaying highly stable output for a long test duration of over 12,000 cycles with less than $5 \%$ variation and in conjunction with a fly-buck circuit was able to power simple electronic devices The work, therefore, provides a facile route to develop flexible, metal-free TENGs and 
concurrently provides mechanism to enhance their power density by eliminating the highly resistive interface. When used in conjunction with other optimisation techniques, the principles of direct synthesis of LIG electrodes on triboelectric surfaces can provide a simple, costeffective route for rapidly expanding the role and uptake of TENG energy harvesters.

\section{References:}

[1] M. Chan, D. Estève, J.-Y. Fourniols, C. Escriba, E. Campo, Smart wearable systems: Current status and future challenges, Artif. Intell. Med. 56 (2012) 137-156. https://doi.org/10.1016/j.artmed.2012.09.003.

[2] J. Gubbi, R. Buyya, S. Marusic, M. Palaniswami, Internet of Things (IoT): A vision, architectural elements, and future directions, Future Gener. Comput. Syst. 29 (2013) 1645-1660. https://doi.org/10.1016/j.future.2013.01.010.

[3] M.A. Hannan, S. Mutashar, S.A. Samad, A. Hussain, Energy harvesting for the implantable biomedical devices: issues and challenges, Biomed. Eng. OnLine. 13 (2014) 79. https://doi.org/10.1186/1475-925X-13-79.

[4] H. Askari, A. Khajepour, M.B. Khamesee, Z. Saadatnia, Z.L. Wang, Piezoelectric and triboelectric nanogenerators: Trends and impacts, Nano Today. 22 (2018) 10-13. https://doi.org/10.1016/j.nantod.2018.08.001.

[5] C. Wu, A.C. Wang, W. Ding, H. Guo, Z.L. Wang, Triboelectric Nanogenerator: A Foundation of the Energy for the New Era, Adv. Energy Mater. 9 (2019) 1802906. https://doi.org/10.1002/aenm.201802906.

[6] X.-S. Zhang, M.-D. Han, B. Meng, H.-X. Zhang, High performance triboelectric nanogenerators based on large-scale mass-fabrication technologies, Nano Energy. 11 (2015) 304-322. https://doi.org/10.1016/j.nanoen.2014.11.012.

[7] M.M. Apodaca, P.J. Wesson, K.J.M. Bishop, M.A. Ratner, B.A. Grzybowski, Contact Electrification between Identical Materials, Angew. Chem. Int. Ed. 49 (2010) 946-949. https://doi.org/10.1002/anie.200905281.

[8] S. Niu, S. Wang, L. Lin, Y. Liu, Y.S. Zhou, Y. Hu, Z.L. Wang, Theoretical study of contact-mode triboelectric nanogenerators as an effective power source, Energy Environ. Sci. 6 (2013) 3576. https://doi.org/10.1039/c3ee42571a.

[9] L. Dhakar, P. Pitchappa, F.E.H. Tay, C. Lee, An intelligent skin based self-powered finger motion sensor integrated with triboelectric nanogenerator, Nano Energy. 19 (2016) 532 540. https://doi.org/10.1016/j.nanoen.2015.04.020.

[10] S. Lee, Y. Lee, D. Kim, Y. Yang, L. Lin, Z.-H. Lin, W. Hwang, Z.L. Wang, Triboelectric nanogenerator for harvesting pendulum oscillation energy, Nano Energy. 2 (2013) 11131120. https://doi.org/10.1016/j.nanoen.2013.08.007.

[11] N. Soin, P. Zhao, K. Prashanthi, J. Chen, P. Ding, E. Zhou, S.C. Ray, C. Tsonos, T. Thundat, E. Siores, J. Luo, High performance triboelectric generators based on phaseinversion membranes of poly(vinylidene fluoride)-zinc stannate (PVDF-ZnSnO3) and $\begin{array}{llllll}\text { polyamide-6 (PA6), Nano Energy } & 30 & \text { (2016), } & \text { 470-480. }\end{array}$ https://doi.org/10.1016/j.nanoen.2016.10.040

[12] P. Zhao, N. Soin, K. Prashanthi, J. Chen, S. Dong, E. Zhou, Z. Zhu, A.A. Narasimulu, C.D. Montemagno, L. Yu, J. Luo, Emulsion Electrospinning of Polytetrafluoroethylene (PTFE) Nanofibrous Membranes for High-Performance Triboelectric Nanogenerators, ACS Appl. Mater. Interfaces. $10 \quad$ (2018) 5880-5891. 
https://doi.org/10.1021/acsami.7b18442.

[13] C. Han, C. Zhang, W. Tang, X. Li, Z.L. Wang, High power triboelectric nanogenerator based on printed circuit board (PCB) technology, Nano Research 8 (2015) 722-730. https://doi.org/10.1007/s12274-014-0555-3.

[14] L. Shi, S. Dong, P. Ding, J. Chen, S. Liu, S. Huang, H. Xu, U. Farooq, S. Zhang, S. Li, J. Luo, Carbon electrodes enable flat surface PDMS and PA6 triboelectric nanogenerators to achieve significantly enhanced triboelectric performance. Nano Energy, 55 (2019), 548-557. https://doi.org/10.1016/j.nanoen.2018.11.012

[15] N. Inagaki, S. Tasaka, A. Onodera, Improved adhesion between Kapton film and copper metal by silane-coupling reactions, J. Appl. Polym. Sci. 73(1999), 1645-1654. https://onlinelibrary.wiley.com/doi/abs/10.1002/(SICI)1097-4628(19990829)

[16] H. Hwang, K.Y. Lee, D. Shin, J. Shin, S. Kim, W. Choi, Metal-free, flexible triboelectric generator based on MWCNT mesh film and PDMS layers, Appl. Surf. Sci. 442 (2018) 693-699. https://doi.org/10.1016/j.apsusc.2018.02.227.

[17] Y. Yang, N. Sun, Z. Wen, P. Cheng, H. Zheng, H. Shao, Y. Xia, C. Chen, H. Lan, X. Xie, C. Zhou. Liquid-metal-based super-stretchable and structure-designable triboelectric nanogenerator for wearable electronics. ACS Nano, 12(2)(2018), 2027-2034. https://doi.org/10.1021/acsnano.8b00147

[18] X. Xia, J. Chen, G. Liu, M. S. Javed, X. Wang, C. Hu, Aligning graphene sheets in PDMS for improving output performance of triboelectric nanogenerator, Carbon. 111 (2017) 569-576. https://doi.org/10.1016/j.carbon.2016.10.041.

[19] M. Schumann, R. Sauerbrey, M.C. Smayling, Permanent increase of the electrical conductivity of polymers induced by ultraviolet laser radiation, Appl. Phys. Lett. 58 (1991) 428-430. https://doi.org/10.1063/1.104624.

[20] J. Lin, Z. Peng, Y. Liu, F. Ruiz-Zepeda, R. Ye, E.L.G. Samuel, M.J. Yacaman, B.I. Yakobson, J.M. Tour, Laser-induced porous graphene films from commercial polymers, Nat. Commun. 5 (2014) 5714. https://doi.org/10.1038/ncomms6714.

[21] Y. Chyan, R. Ye, Y. Li, S.P. Singh, C.J. Arnusch, J.M. Tour, Laser-Induced Graphene by Multiple Lasing: Toward Electronics on Cloth, Paper, and Food, ACS Nano. 12 (2018) 2176-2183. https://doi.org/10.1021/acsnano.7b08539.

[22] M.G. Stanford, J.T. Li, Y. Chyan, Z. Wang, W. Wang, J.M. Tour, Laser-Induced Graphene Triboelectric Nanogenerators, ACS Nano. 13 (2019) 7166-7174. https://doi.org/10.1021/acsnano.9b02596.

[23] H. Chu, H. Jang, Y. Lee, Y. Chae, J.-H. Ahn, Conformal, graphene-based triboelectric nanogenerator for self-powered wearable electronics, Nano Energy. 27 (2016) 298-305. https://doi.org/10.1016/j.nanoen.2016.07.009.

[24] J. Luo, F. R. Fan, T. Jiang, Z. Wang, W. Tang, C. Zhang, M. Liu, G. Cao, Z. L. Wang. Integration of micro-supercapacitors with triboelectric nanogenerators for a flexible selfcharging power unit. Nano Research, 8(12)(2015), 3934-3943. https://doi.org/10.1007/s12274-015-0894-8

[25] G. Bhattacharya, S. J. Fishlock, A. Pritam, S. S. Roy, J. A. McLaughlin, Recycled Red Mud - Decorated Porous 3D Graphene for High - Energy Flexible Micro Supercapacitor. Adv. Sust. Sys., accepted (2020), 1900133. https://doi.org/10.1002/adsu.201900133

[26] X. Ruan, R. Wang, J. Luo, Y. Yao, T. Liu. Experimental and modeling study of CO2 laser writing induced polyimide carbonization process. Materials \& Design, 160(2018), 11681177. https://doi.org/10.1016/j.matdes.2018.10.050

[27] S. Luo, P. T. Hoang, T. Liu. Direct laser writing for creating porous graphitic structures and their use for flexible and highly sensitive sensor and sensor arrays. Carbon, 96(2016), 522-531. https://doi.org/10.1016/j.carbon.2015.09.076 
[28] M. Inagaki, N. Ohta, Y. Hishiyama. Aromatic polyimides as carbon precursors. Carbon, 61(2013), 1-21. https://doi.org/10.1016/j.carbon.2013.05.035

[29] M. Inagaki, S. Harada, T. Sato, T. Nakajima, Y. Horino, K. Morita. Carbonization of polyimide film "Kapton". Carbon, 27(2)(1989), 253-257. https://doi.org/10.1016/00086223(89)90131-0

[30] J. Rodrigues, J. Zanoni, G. Gaspar, A. J. Fernandes, A. F. Carvalho, N. F. Santos, T. Monteiro, F. M. Costa. ZnO decorated laser-induced graphene produced by direct laser scribing. $\quad$ Nanoscale Advances, $\quad 1(8)(2019), \quad 3252-3268$ https://doi.org/10.1039/c8na00391b

[31] N. Soin, S. S. Roy, C. O'Kane, J. A. McLaughlin, T. H. Lim, C. J. Hetherington. Exploring the fundamental effects of deposition time on the microstructure of graphene nanoflakes by Raman scattering and X-ray diffraction. Cryst. Engg. Comm., 13(1)(2011), 312-318. https://doi.org/10.1039/C0CE00285B

[32] R. Ye, C. Xiang, J. Lin, Z. Peng, K. Huang, Z. Yan, N. P. Cook, E. L. Samuel, C. C. Hwang, G. Ruan, G. Ceriotti. Coal as an abundant source of graphene quantum dots. Nat. Comm., 4(2013), 2943. https://doi.org/10.1038/ncomms3943

[33] R. Ye, Y. Chyan, J. Zhang, Y. Li, X. Han, C. Kittrell, J. M. Tour. Laser - induced graphene formation on wood. Adv. Mater., 29(37)(2017), 1702211. https://doi.org/10.1002/adma.201702211

[34] A. Gupta, L. Holoidovsky, C. Thamaraiselvan, A. K. Thakur, S. P. Singh, M. M. Meijler, C. J. Arnusch. Silver-doped laser-induced graphene for potent surface antibacterial activity and anti-biofilm action. Chem. Comm., 55(48)(2019), 6890-6893. https://doi.org/10.1039/C9CC02415H

[35] A. Lamberti, F. Perrucci, M. Caprioli, M. Serrapede, M. Fontana, S. Bianco, S. Ferrero, E. Tresso. New insights on laser-induced graphene electrodes for flexible supercapacitors: tunable morphology and physical properties. Nanotechnology, 28(17)(2017), 174002 https://doi.org/10.1088/1361-6528/aa6615

[36] D. W. Zeng, K.C. Yung, C. S. Xie. XPS investigation of the chemical characteristics of Kapton films ablated by a pulsed TEA CO2 laser. Surf. Coat. Tech., 153(2-3)(2002), 210216. https://doi.org/10.1016/S0257-8972(01)01696-6

[37] S. Lee, S. Jeon. Laser-Induced Graphitization of Cellulose Nanofiber Substrates under Ambient Conditions. ACS Sust. Chem. Engg., 7(2)(2018), 2270-2275. https://doi.org/10.1021/acssuschemeng.8b04955

[38] A. A. Narasimulu, P. Zhao, N. Soin, K. Prashanthi, P. Ding, J. Chen, S. Dong, L. Chen, E. Zhou, C. D. Montemagno, J. Luo. Significant triboelectric enhancement using interfacial piezoelectric $\mathrm{ZnO}$ nanosheet layer. Nano Energy, 40(2017), 471-480. https://doi.org/10.1016/j.nanoen.2017.08.053

[39] P. Zhao, N. Soin, A. Kumar, L. Shi, S. Guan, C. Tsonos, Z. Yu, S. C. Ray, J. A. McLaughlin, Z. Zhu, E. Zhou, J. Luo. Expanding the portfolio of tribo-positive materials: Aniline formaldehyde condensates for high charge density triboelectric nanogenerators. Nano Energy, 67(2020), 104291. https://doi.org/10.1016/j.nanoen.2019.104291

[40] P. Ding, J. Chen, U. Farooq, P. Zhao, N. Soin, L. Yu, H. Jin, X. Wang, S. Dong, J. Luo Realizing the potential of polyethylene oxide as new positive tribo-material: Over 40 $\mathrm{W} / \mathrm{m} 2$ high power flat surface triboelectric nanogenerators. Nano energy, 46(2018), 6372. https://doi.org/10.1016/j.nanoen.2018.01.034

[41] M. Taghavi, L. Beccai, A contact-key triboelectric nanogenerator: Theoretical and experimental study on motion speed influence, Nano Energy. 18 (2015) 283-292. https://doi:10.1016/j.nanoen.2015.10.019.

[42] C. Zhang, J. Chen, W. Xuan, S. Huang, B. You, W. Li, L. Sun, H. Jin, X. Wang, S. Dong, J. Luo. Conjunction of triboelectric nanogenerator with induction coils as wireless power 
sources and self-powered wireless sensors. Nat. Comm., 11(1), pp.1-10. https://doi.org/10.1038/s41467-019-13653-w

[43] A.F. Diaz, R.M. Felix-Navarro, A semi-quantitative tribo-electric series for polymeric materials: the influence of chemical structure and properties, J. Electrost. 62 (2004) 277 290. https://doi.org/10.1016/j.elstat.2004.05.005.

[44] H. Zou, Y. Zhang, L. Guo, P. Wang, X. He, G. Dai, H. Zheng, C. Chen, A.C. Wang, C. $\mathrm{Xu}, \mathrm{Z} . \mathrm{L}$. Wang, Quantifying the triboelectric series, Nat. Commun. 10 (2019) 1427. https://doi.org/10.1038/s41467-019-09461-x

[45] J. Peng, S. D. Kang, G. J. Snyder. Optimization principles and the figure of merit for

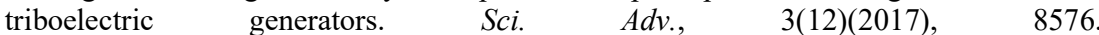
https://doi.org/10.1126/sciadv.aap8576

[46] C. Xu, B. Zhang, A. C. Wang, W. Cai, Y. Zi, P. Feng, Z. L. Wang. Effects of Metal Work Function and Contact Potential Difference on Electron Thermionic Emission in Contact Electrification. Adv. Func. Mater., 29(29)(2019), 1903142. https://doi.org/10.1002/adfm.201903142

[47] Y. Zhang, S. Ke, H. Huang, L. Zhao, L. Yu, H. L. Chan. Dielectric relaxation in polyimide nanofoamed films with low dielectric constant. Appl. Phys. Lett., 92(5)(2008), 052910. https://doi.org/10.1063/1.2840715

[48] J. R. Macdonald. Comments on the electric modulus formalism model and superior alternatives to it for the analysis of the frequency response of ionic conductors. J. Phys. Chem. Solids, 70(3-4)(2009), 546-554. https://doi.org/10.1016/j.jpcs.2008.12.012

[49] K. Yamamoto, H. Namikawa. Conduction current relaxation of inhomogeneous conductor I. Jpn. J. Appl. Phys., 27(10R)(1988), 1845 https://doi.org/10.1143/JJAP.27.1845

[50] C. Tsonos, L. Apekis, K. Viras, L. Stepanenko, L. Karabanova, L. Sergeeva. Electrical and dielectric behavior in blends of polyurethane-based ionomers. Solid State Ionics, 143(2)(2001), 229-249. https://doi.org/10.1016/S0167-2738(01)00858-X

[51] P.B. Macedo, C.T. Moynihan, R. Bose. The Role of Ionic Diffusion in Polarization in Vitreous Ionic Conductors, Phys. Chem. Glasses, 13(6)(1972), 171-179.

[52] A. Delgado, M. F. García-Sánchez, J. C. M'Peko, A. R. Ruiz-Salvador, G. RodríguezGattorno, Y. Echevarría, F. Fernández-Gutierrez. An elementary picture of dielectric spectroscopy in solids: Physical basis. J. Chem. Edu., 80(9)(2003), 1062. https://doi.org/10.1021/ed080p1062 


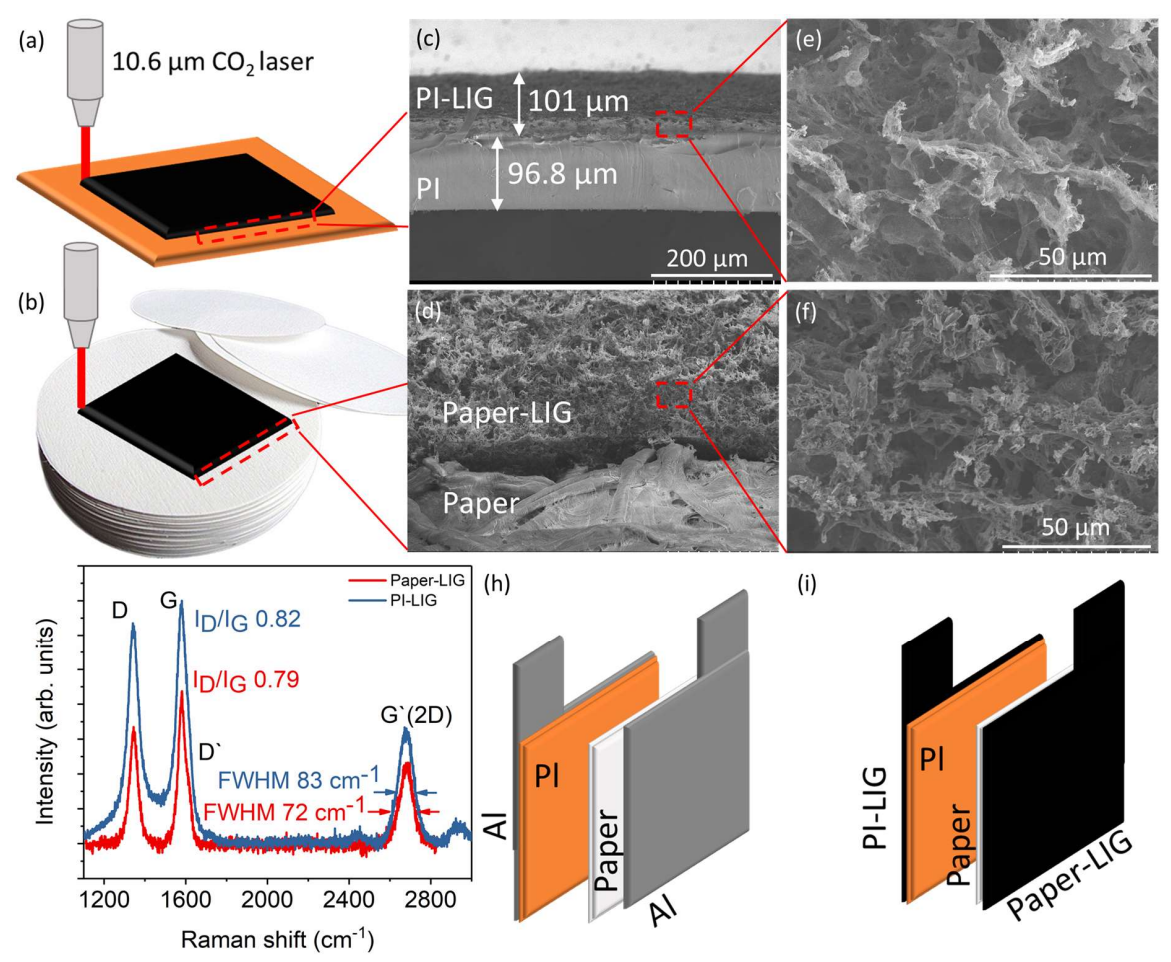

Fig. 1. (a, b) Illustration of the PI and paper substrates showing the formation of LIG (PI-LIG, paper-LIG) films through laser irradiation. The corresponding cross-section and surface morphology SEM images are shown in (c, e) for PI-LIG and (d, f) paper-LIG samples, respectively. (g,h ) illustration of the PI-Paper TENGS with aluminium and LIG electrodes. 

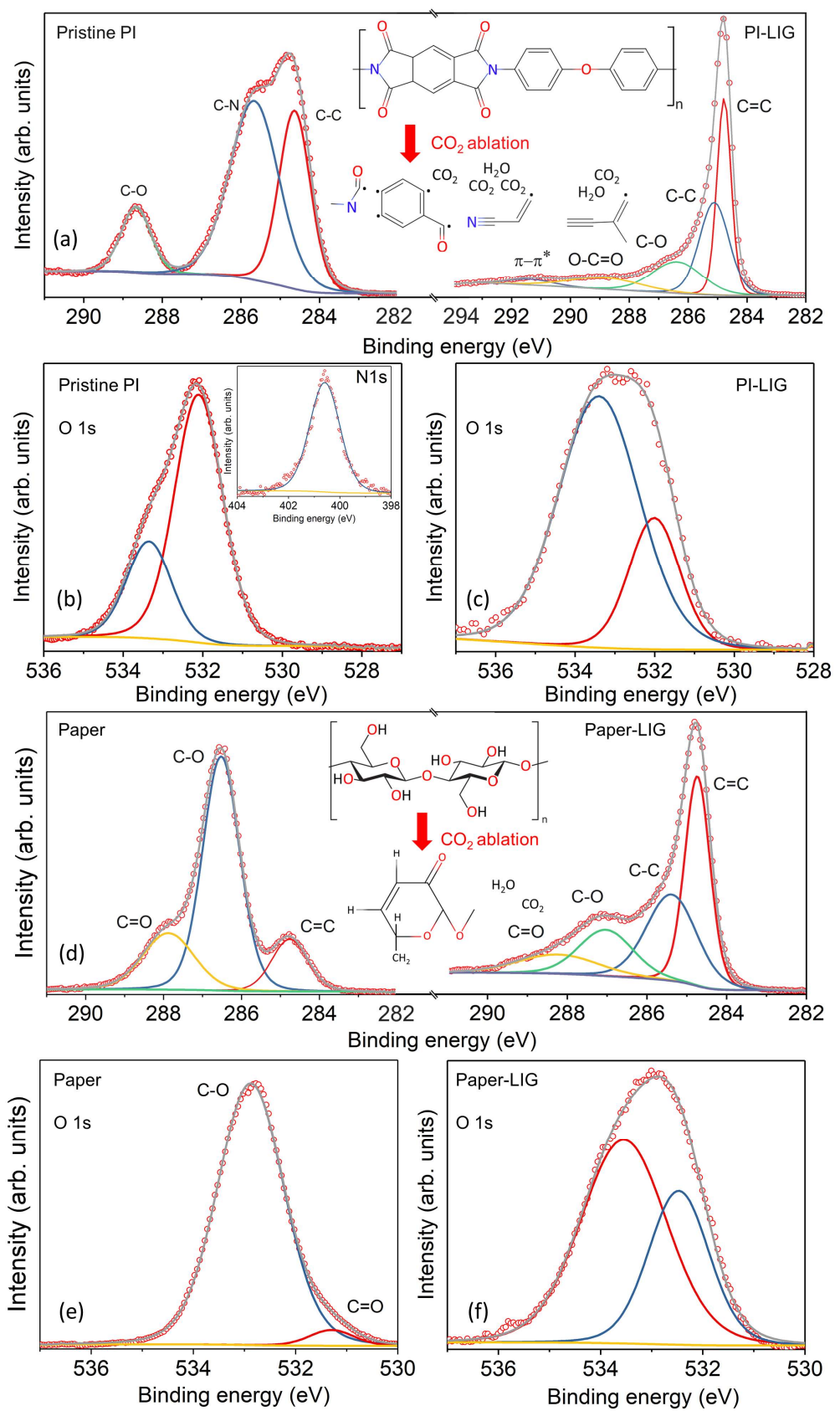

Fig. 2: (a) The variation of the C 1s XPS core-level spectra for pristine PI (left panel) which 
changes significantly upon lasing (inset) confirming the formation of PI-LIG (right panel). The O 1s core level spectra for (b) pristine PI and (c) PI-LIG samples. (c) The changes occurring in the $\mathrm{C} 1 \mathrm{~s}$ core level spectra when the pristine paper substrate (left panel) is converted to paperLIG (right panel) upon $\mathrm{CO}_{2}$ lasing process (inset). $\mathrm{C} 1 \mathrm{~s}$ and $\mathrm{O} 1 \mathrm{~s}$ spectra for paper substrate and paper-LIG are shown in (e, f) and $(\mathrm{g}, \mathrm{h})$, respectively. 

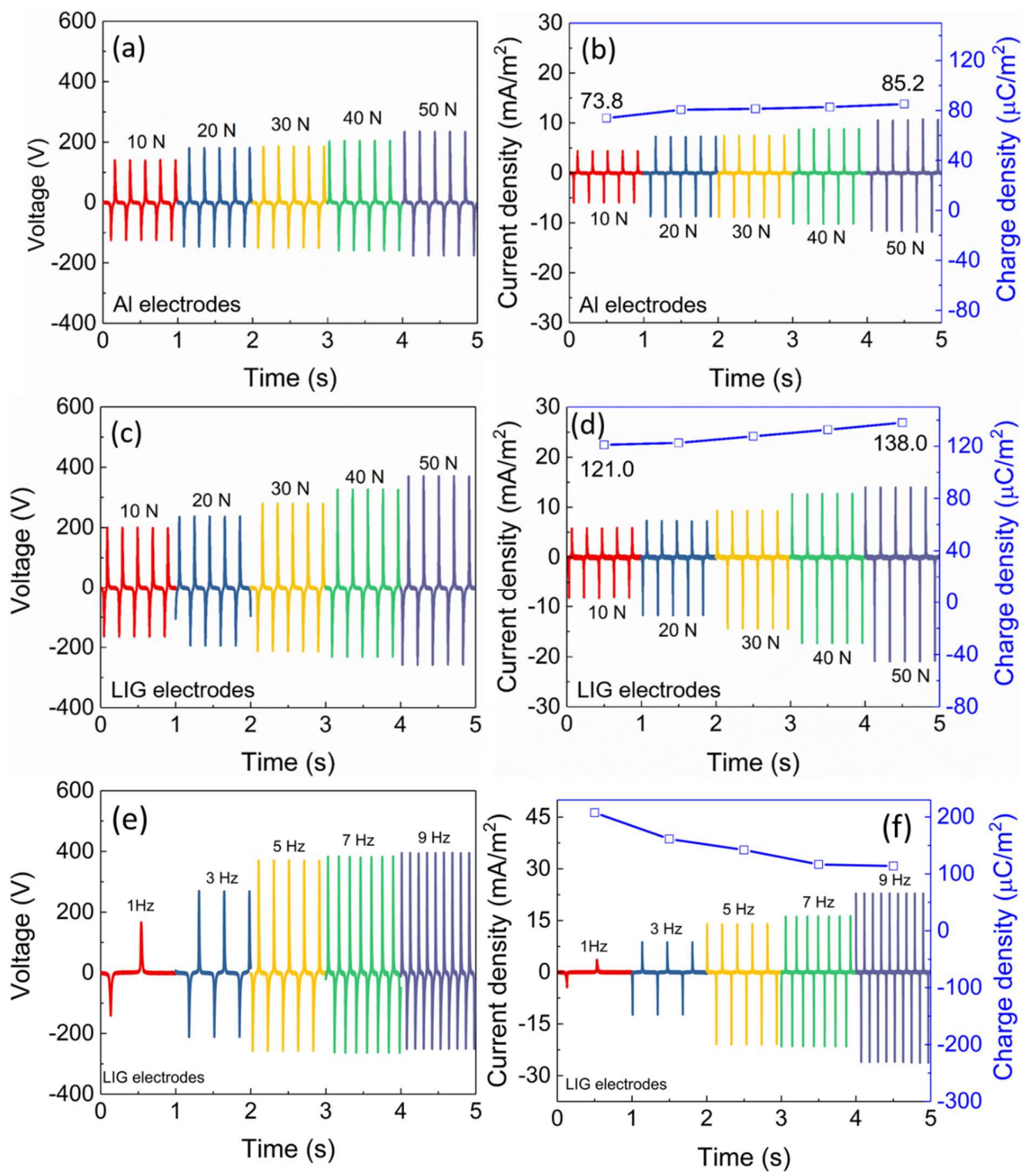

Fig. 3. Electrical measurement results of the paper/PI TENG with $(a, b)$ aluminium electrodes and (c, d) LIG electrodes at various impact force ranging from 10 to $50 \mathrm{~N}$. The corresponding charge density is obtained from the integral of the area under the curve for the current response. The PI-paper LIG TENGs were subjected to mechanical excitation at varying frequencies from 1-9 Hz with corresponding (e) voltage and (f) current density and charge density measurements, respectively. 

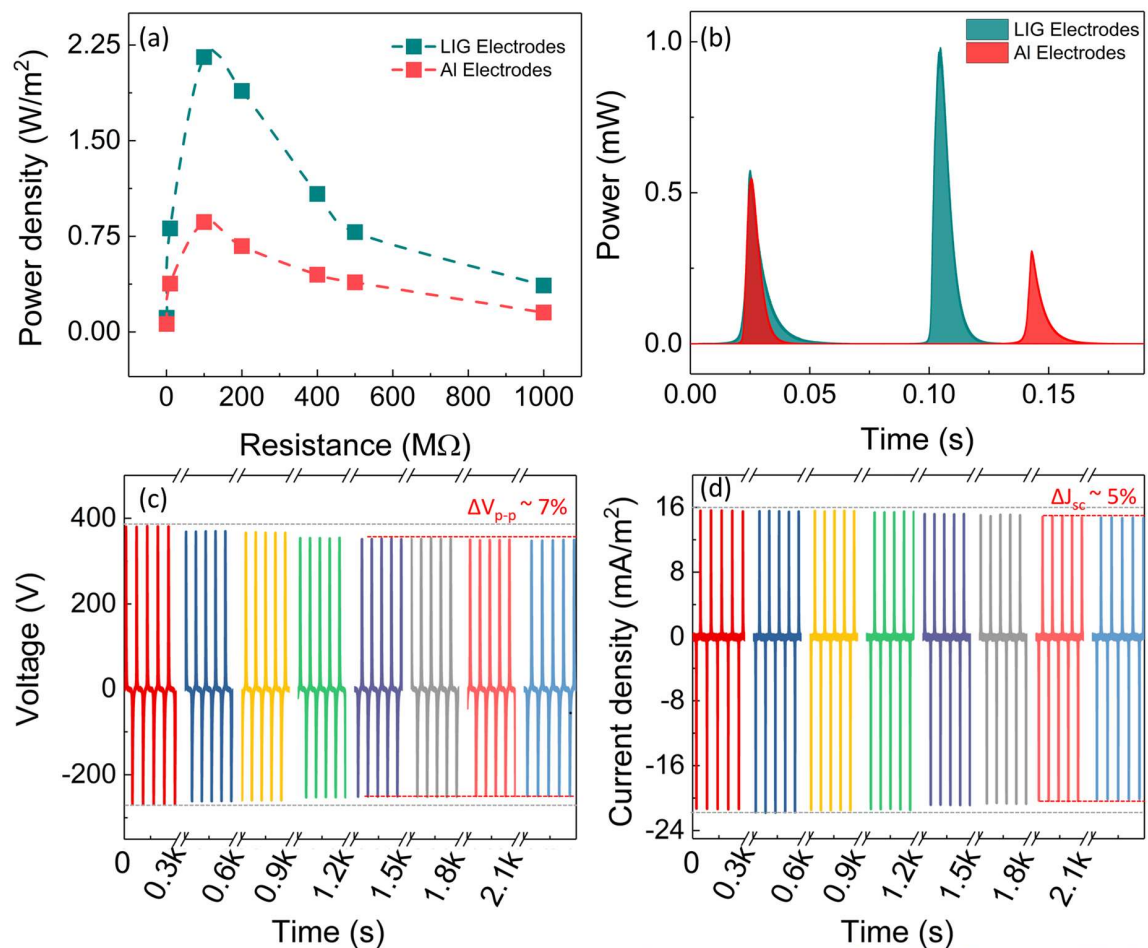

(e)
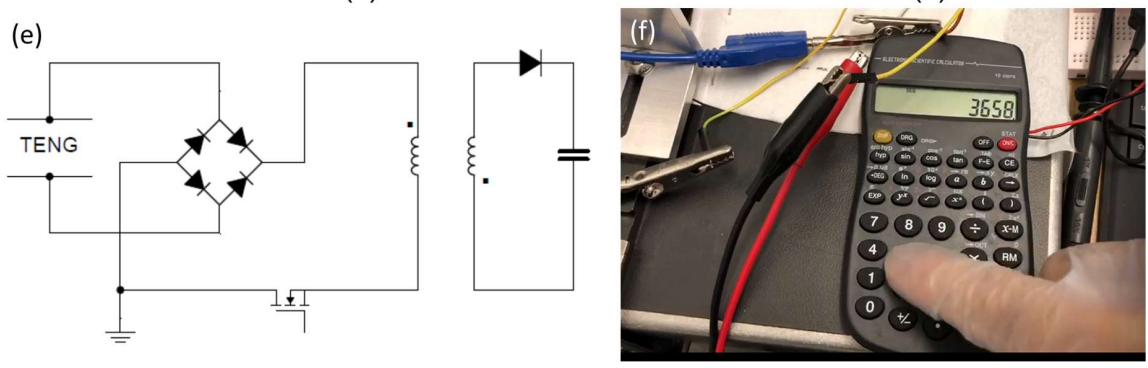

Fig. 4. (a) Power density values measured with variable load resistance for the paper/PI-LIG and paper/PI-Al TENGs, (b) the power output of a single energy generation cycle at the impedance matching condition (100 M $\Omega$ ). The paper/PI-LIG TENG shows consistent (c) voltage output with a $\Delta \mathrm{V}_{\mathrm{p}-\mathrm{p}} \sim 7 \%$ and (d) current density output (with $\Delta \mathrm{J}_{\mathrm{sc}} \sim 5 \%$ ) behaviour for the test duration of 12,000 energy generation cycles at a fixed working frequency of $5 \mathrm{~Hz}$. 

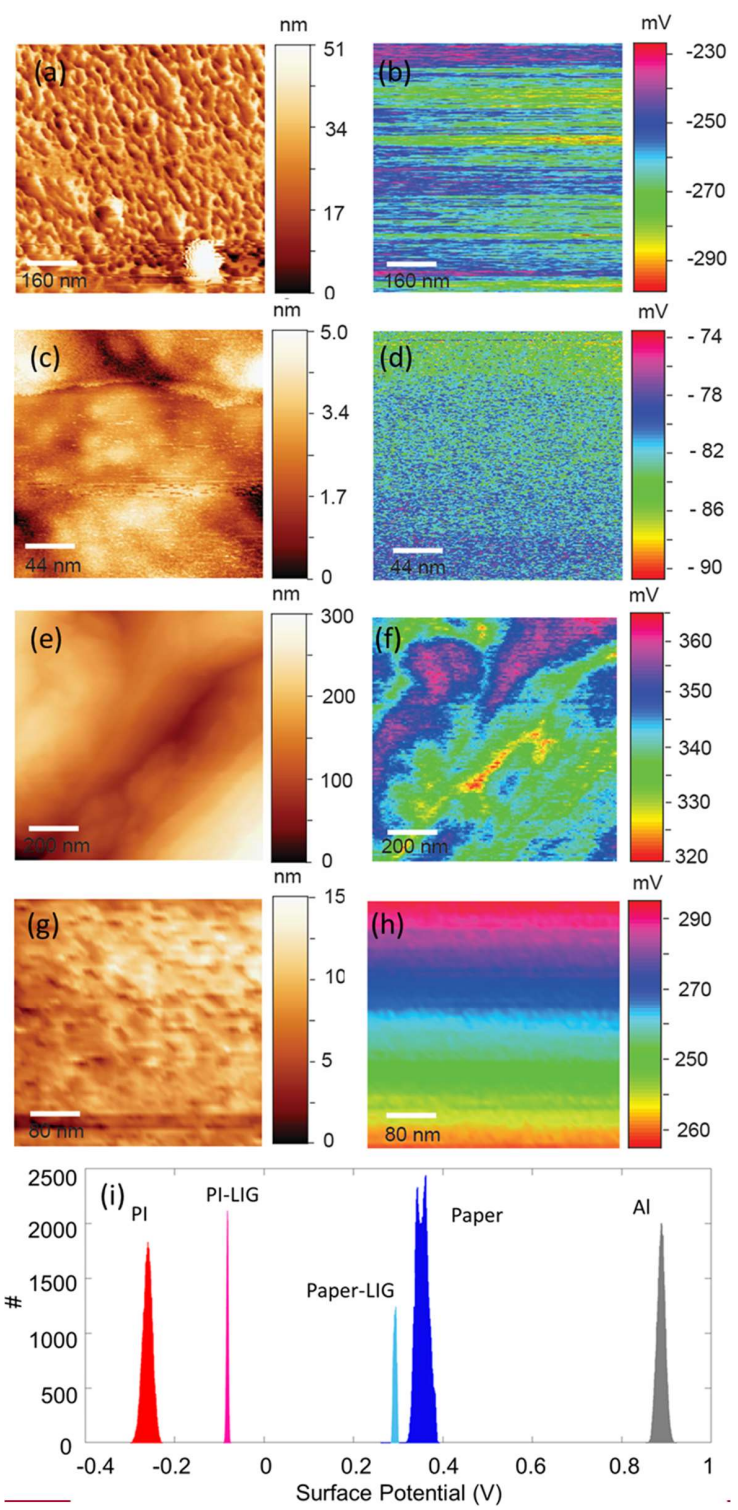

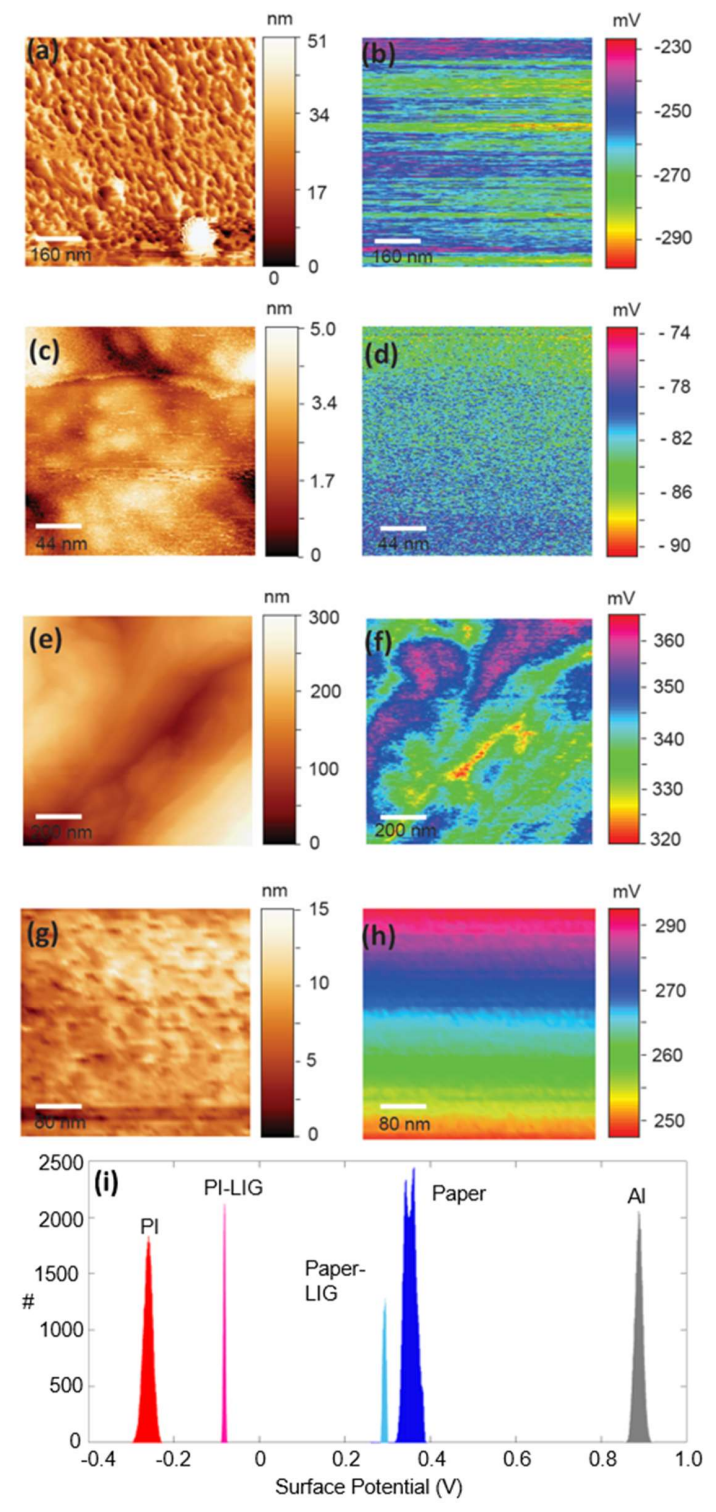

Fig. 5: The topography and surface potential maps for (a, b) pristine PI; (c, d) PI-LIG synthesised from pristine PI; (e, f) pristine cellulosic paper; $(\mathrm{g}, \mathrm{h})$ paper-LIG synthesised from paper treated with a fire-retardant, respectively; (i) the histogram of distribution of the surface potentials of the pristine Al, PI, paper and the subsequent PI-LIG and paper-LIG substrates.

Commented [AK1]: The figure has a problem in panel (h). On scale bar, 250 came above $260 \ldots$ I have inserted a new figure which addresses this issue. Please check if the quality of new figure 5 inserted here is reasonable. Else, please edit the way you had done it before using figure_KPFM (corrected now) 

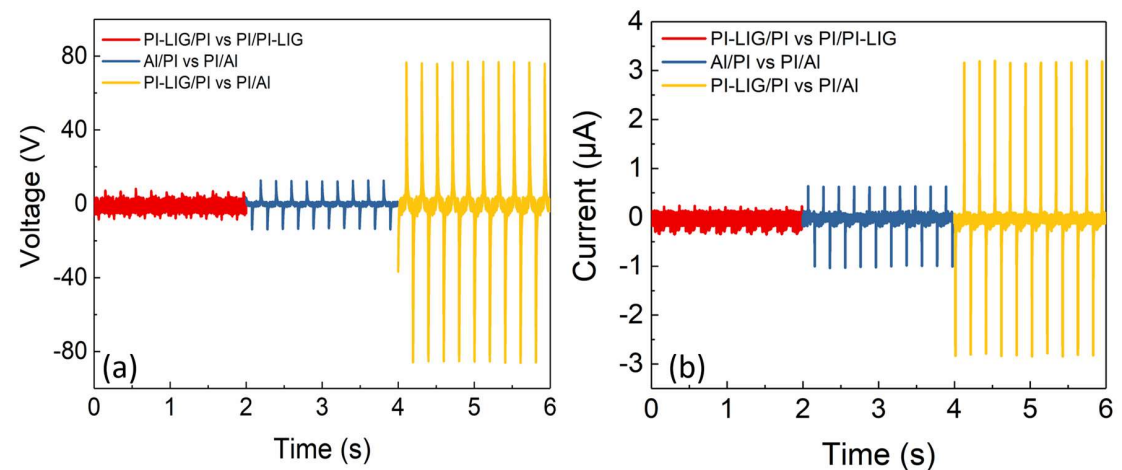

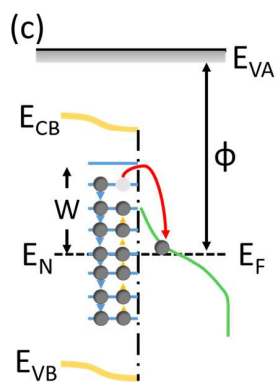

Dielectric Metal (d)

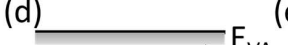

(e)

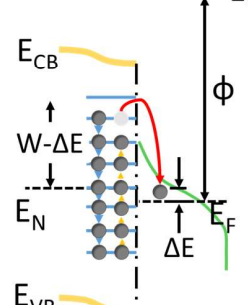

Dielectric Metal
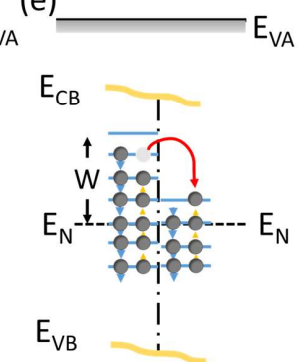

Dielectric Dielectric (f)

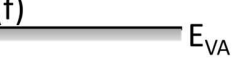

$\mathrm{E}_{\mathrm{CB}}-\mathrm{I}$

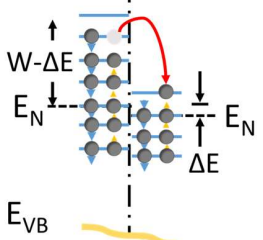

Dielectric Dielectric

Fig. 6: Electrical measurement results (a) voltage output and (b) peak current values of the PI/PI TENG with various combination of electrodes at a fixed force of $50 \mathrm{~N}$ and $5 \mathrm{~Hz}$ frequency confirming the role of electrodes in dictating the electrical output from similar triboelectric materials. A depiction of surface state models for visualising of the regulation of potential barrier height of materials during triboelectrification process is shown in (c-f). Charge transfer between a dielectric (with initial negative charge) and conductor when the neutral level of surface states, $E_{N}$ of the former is (c) as high as, (d) higher than of the latter. Charge transfer between dielectric $\mathrm{A}$ (with initial negative charges) and dielectric $\mathrm{B}$ when $\mathrm{E}_{\mathrm{N}}$ of the former is (e) as high as, (f) higher than that of the latter. $E_{V A}$, vacuum level; $E_{C B}$, conduction band; $E_{V B}$, valence band; W, potential barrier; $\Phi$, work function; and $E_{F}$, Fermi level. ${ }^{[48]}$ 

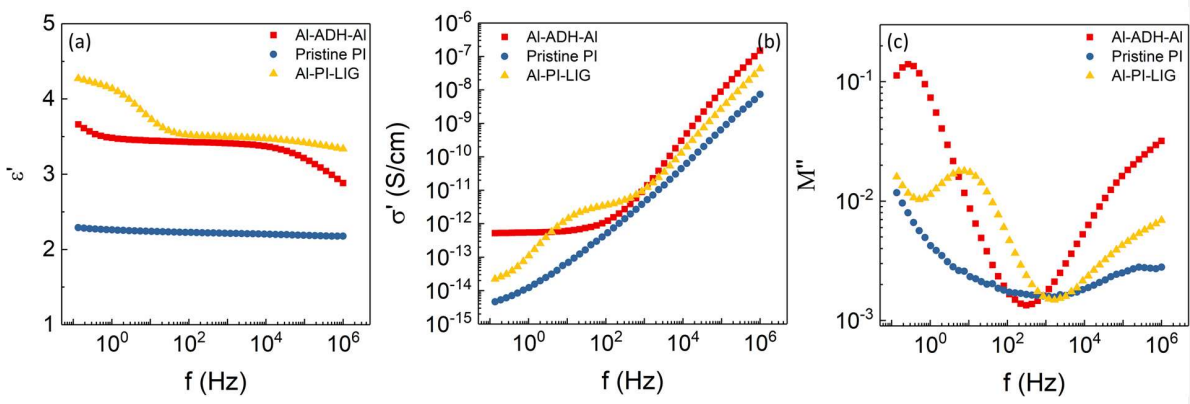

Fig. 7: (a) The variation of the dielectric permitivitty $\varepsilon$ ' with the corresponding changes in the (b) AC electrical conductivity and (c) dependence of imaginary part of electric modulus as a function of frequency, for of the Al-adhesive-Al, pristine PI and Al-PI-(PI-LIG) combinations at room temperature. 